\title{
Key advances: translation and location
}

Each February we chronicle the previous year's advances in clinical oncology with our Year-in-Review articles, which we collect across the entirety of the eight Nature Reviews clinical titles to provide the Key Advances in Medicine e-book (soon available via our journal homepage). Unsurprisingly, in oncology, immunotherapy featured prominently among the game-changing advances made in 2016.

In head and neck cancer, immunotherapy not only prolonged responses and overall survival, but in one study also improved quality of life. Progress in radiotherapy dose de-escalation, as well as in the use of PETCT imaging to reduce invasive surgeries and associated complications, are other key advances in this disease.

Studies in patients with sarcoma began to unravel the extent of molecular heterogeneity and define distinct molecular subtypes that will pave the way for better classification to help improve personalization of treatment. In particular, regimens containing olaratumab, eribulin or regorafenib were tested in patient groups, providing new options for this difficult-to-study cancer.

In small-cell lung cancer (SCLC), basic research in mouse models elegantly demonstrated roles for Nfib and Mycl as drivers of metastasis, illustrating how mechanisms of disease development can be teased apart - an admirable advance, especially in these rare tumours. Translational progress in SCLC was also highlighted by the clinical activity of rovalpituzumab (an antibodydrug conjugate targeting the Notch ligand DLL3), with impressive response and 1-year survival rates reported in the recurrent metastatic setting.

For patients with neuroendocrine tumours (NETs), of the lung or gastrointestinal tract, not only did everolimus cement its place in the treatment landscape, but a pivotal study from MSKCC distinguished well-differentiated and poorly differentiated subtypes of high-grade NETs, enabling practical guidelines for their classification.

The dance of the doublet or triplet therapies in multiple myeloma was concluded with data showing efficacy of bortezomib, lenalidomide and dexamethazone in newly-diagnosed patients. Daratumumab emerged as an important new treatment for patients with relapsed disease, with triplet regimens containing this agent providing durable remissions in this setting, with further progress expected with a range of novel regimens.

Finally, in renal-cell carcinoma, a conclusive benefit from adjuvant sunitinib therapy was not established. In the second-line metastatic setting, however, both cabozantinib and nivolumab were shown to improve overall survival and quality of life, compared with everolimus.
We'd also like to draw attention to our online correspondence article by Cremolini et al., which eloquently describes advances made in 2016 regarding the role of tumour location (right or left sideness) as a key prognostic factor and predictor of benefit from anti-EGFR or anti-VEGF therapy for colorectal cancer (CRC). Clear evidence now shows that patients with CRC have a significantly worse prognosis if the tumour is right-sided (located up to the proximal two-thirds of the transverse colon) versus left-sided (located within the distal third of the transverse colon or beyond), independent of disease stage. Patients with left-sided tumours derive an overall survival benefit from anti-EGFR therapy, an association notably absent in patients with right-sided tumours; multiagent chemotherapy plus bevacizumab (an antiVEGF agent) seems to be more efficacious in those with right-sided tumours.

Importantly, Cremolini et al. provide an update to the treatment algorithm proposed in their Review published in this journal in 2015, to illustrate the implications of tumour location on allocation of therapy. For example, in patients with $B R A F / R A S$-wild-type tumours, no randomized study has tested whether doublet chemotherapy plus an anti-EGFR antibody or triplet chemotherapy plus bevacizumab is better. Now, Cremolini et al. propose that tumour sideness can guide this decision, with the triplet approach being the preferred option for right-sided tumours, owing to their poor prognosis and resistance to anti-EGFR therapy.

These outcome differences are probably, in part, related to the types of mutations driving left-sided versus right-sided tumours: the former tend to harbour molecular alterations associated with EGFR-dependency, explaining their favourable responsiveness to therapy targeting this pathway, whereas the latter commonly show features of resistance to such treatment. These differences might ultimately arise owing to variations in tumour aetiology related to the anatomy of the colon. Right-sided tumours possibly reside in an altered vascularized region, thereby explaining why anti-VEGF therapies seem to work better against such tumours. Further work is needed to dissect these relationships, but clearly the differences in prognosis and responsiveness to therapies between anatomically distinct colon cancers must go deeper than purely molecular considerations.

Collectively, these advances in translational research and the dedication of trials conducted in the clinical space to better define the best therapy options represent important stratification factors for future studies, and we look forward to seeing what the next year brings! 